\title{
Image Retrieval System based on Colour Shape and Texture Attributes
}

\author{
Prof. Kamble D.R ${ }^{1}$, Davari Ashitosh ${ }^{2}$ \\ Professor, Computer Science \& Engg, DACOE, Karad, India ${ }^{1}$ \\ Student, Computer Science \& Engg, DACOE, Karad, India ${ }^{2}$
}

\begin{abstract}
In general, Image retrieval is well-known research and development field in information management. An image contains several types of visual features which are difficult to extract and combine manually by humans. These papers introduce some visual features of an image: color, shape and texture. There is several method of extraction of an image features and study or learning algorithms for retrieving image. Various methods have been proposed and investigated. These papers introduce, color histogram which is mostly used because of their efficiency, robustness and insensitivity to small changes in camera viewpoint.A color histogram store image's overall color composition. So images with many different appearances can have similar color histogram. Colour histogram is constructed by counting the no of pixels of each color of image. Edges are detected in areas of the image where the intensity level fluctuates sharply, the more rapid the intensity changes the stronger the edge. Texture of each sub-block is obtained by using gray level co-occurrence matrix. A one to one matching scheme is used to compare the query and target image.
\end{abstract}

Keywords: features extraction, histogram, precision.

\section{INTRODUCTION}

Image retrieval system have been developed like IBM QBIC system developed at the IBM Alma den Research Centre, the VIRAGE System developed by the Virage Incorporation, the Photo book System developed by the MIT Media Lab, the Visual Seek system developed at Columbia University, the WBIIS System developed at Stanford University, and the Blob world System developed at U.C. Berkeley.

The common for all of these are to extract signature (color, shape, texture and any other information with which two images could be compared.) for every image based on its pixels values and to define rules for comparing images. Image retrieval systems search through a database to find out images that are perceptually similar to a query image. Nowadays many people give interested in using digital images. So, the size of the image database is grows fast. There is a great need for developing an efficient technique for finding the images.

Image Retrieval approach employs that to search through a database to find images that are perceptually similar to a query image. It is an important to traditional text-based image searching and can greatly enhance the accuracy of the information being returned. It aims to develop an efficient visual-Content-based technique to search.

\section{FEATURE EXTRACTION}

Feature extraction can be referred as extracting beneficial information from images. This information is used as a signature for the image. Each image has its identical feature.

\section{A. Colour:}

The Colour is an important visual feature of an image. The extraction of color feature from images depends on understand and learning the theory of color and the representation of color in images. Image retrieval system uses color histograms for color feature extraction. Colour histogram is constructed by calculating RGB value of each pixel of image. Colour spaces are another important part of relating color to its representation in digital form.

Colour space defined as a model for representing color in terms of intensity value. Colour space defines One to Four dimensional space. A color component or color channel is one of the dimensions. Only three-dimension color spaces use RGB and HSV.

\section{B. Shape :}

Shape feature is more effective in characterizing the content of an image Compared to the other features like texture and color. However, it is a challenging task to accurately extract the shape information from an image. It is a key feature for computer vision applications. However, it is a challenging task to accurately extract the shape information from an image.

The construction of shape descriptors is even more complicated when invariance with respect to a number of possible transformations, such as scaling, shifting and rotation is required. Object contours are invariant to extreme illumination conditions and large variations in texture or color and for some categories shape is more generic than appearance. Automated comparison and grouping of shapes is very often the basis is the areas of 
IARJSET

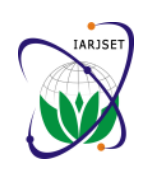

International Advanced Research Journal in Science, Engineering and Technology

National Conference on Innovative Applications and Research in Computer Science and Engineering (NCIARCSE-2017) AGTI's Dr. Daulatrao Aher College Engineering, Vidyanagar Extension, Karad

Vol. 4, Special Issue 4, January 2017

human detection or action recognition. Hence this paper uses shape as a key feature for image retrieval.

\section{Texture:}

The Texture is another important feature of natural images. Because the image can be identified with the color but it may be differ with shape so it plays an important role in extraction. An image can be viewed in a box like structure from which it can get the appearance of the image. Texture represents important information about the structural arrangement of surfaces and their relationship to the surroundings. Many different techniques are developed for analysis texture in past years. Most of the texture features are obtained from the application of a local operator, statistical analysis or measurement in transform domain.

\section{PROPOSED TECHNIQUE}

Image retrieval system searches collection of images based on features that can be extracted from the image files. Images are searched from large logical image database. The logical image representation in image databases system is based on different data modules. An image object is either an entire image or some other meaningful portion of an image. The logical image includes:

1. Colour attributes

2. Shape attributes

3. Texture attributes

IRS system search images based on features that can be extracted from the image files themselves without manual descriptive or indexing labour from humans. The central task in any image data management system is to retrieve image that meet some specified constraints for e.g. find all images that look like this one.

In many areas of business, government, the scholarly world, and doctor's facilities, huge gathering of computerized pictures are being made. A large portion of these systems are result of digitizing existing data of simple photos, graphs, drawing, works of art and prints. For the most part, the main method for looking these was by watchword ordering, or essentially by perusing.

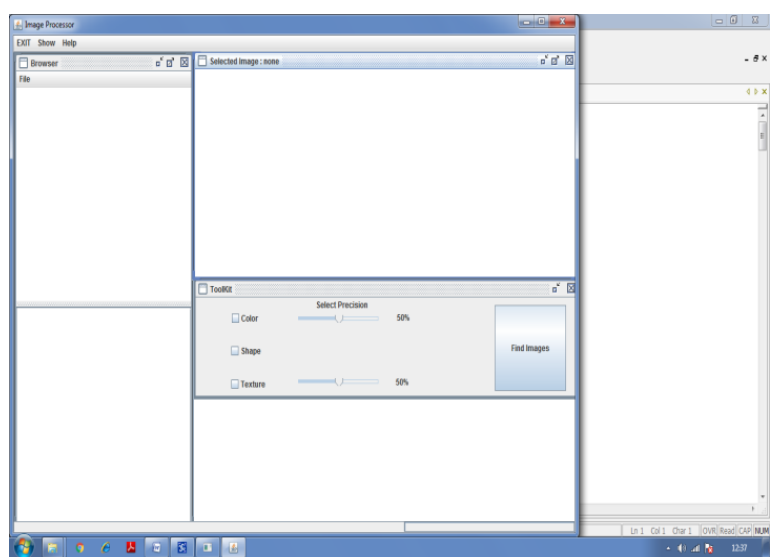

Figure 1.- Main Window in IRS system

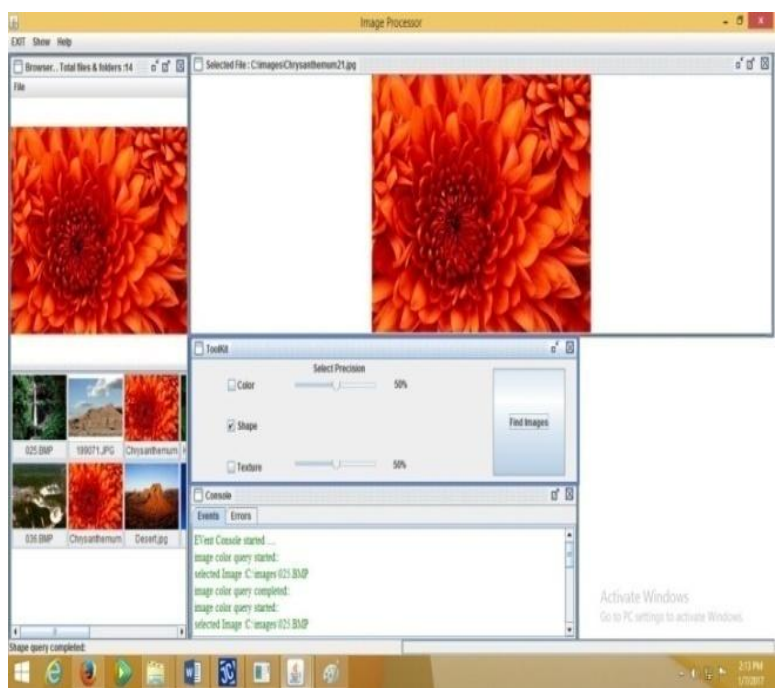

Figure 2.-Selecting shape attribute in IRS system

This is the main window of image retrieval system. Here user has to give query as a input. After giving input to the IRS system user needs to set attributes by which user wants to search the images. Here first figure shows flower image given as an input and shape attribute.

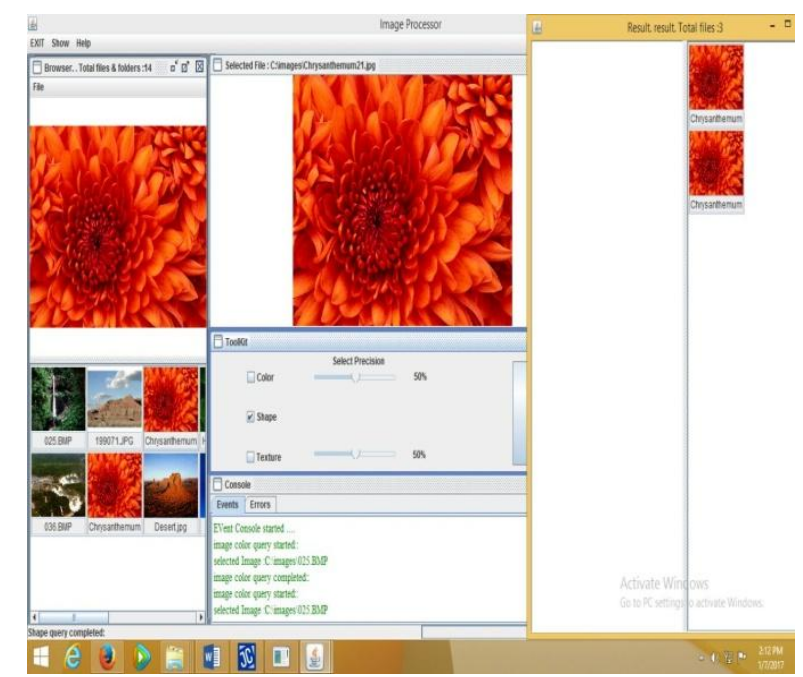

Figure 3. -Result of IRS system based on shape attributes

Figure shows results of a query image searched by shape attribute. IRS system finds two results from its database which are shown in result window.

\section{EXPERIMENTAL RESULT}

Proposed system has been implemented using visual features. In our experiment image database used with different image format. Query image give as an input to the system by selecting attributes. We have performed analysis of different query image by using precision factor and attributes. The accuracy of IRS system can be found out with the help of precision factor. Image can be retrieved by using different attributes for example color, shape and texture. 
IARJSET

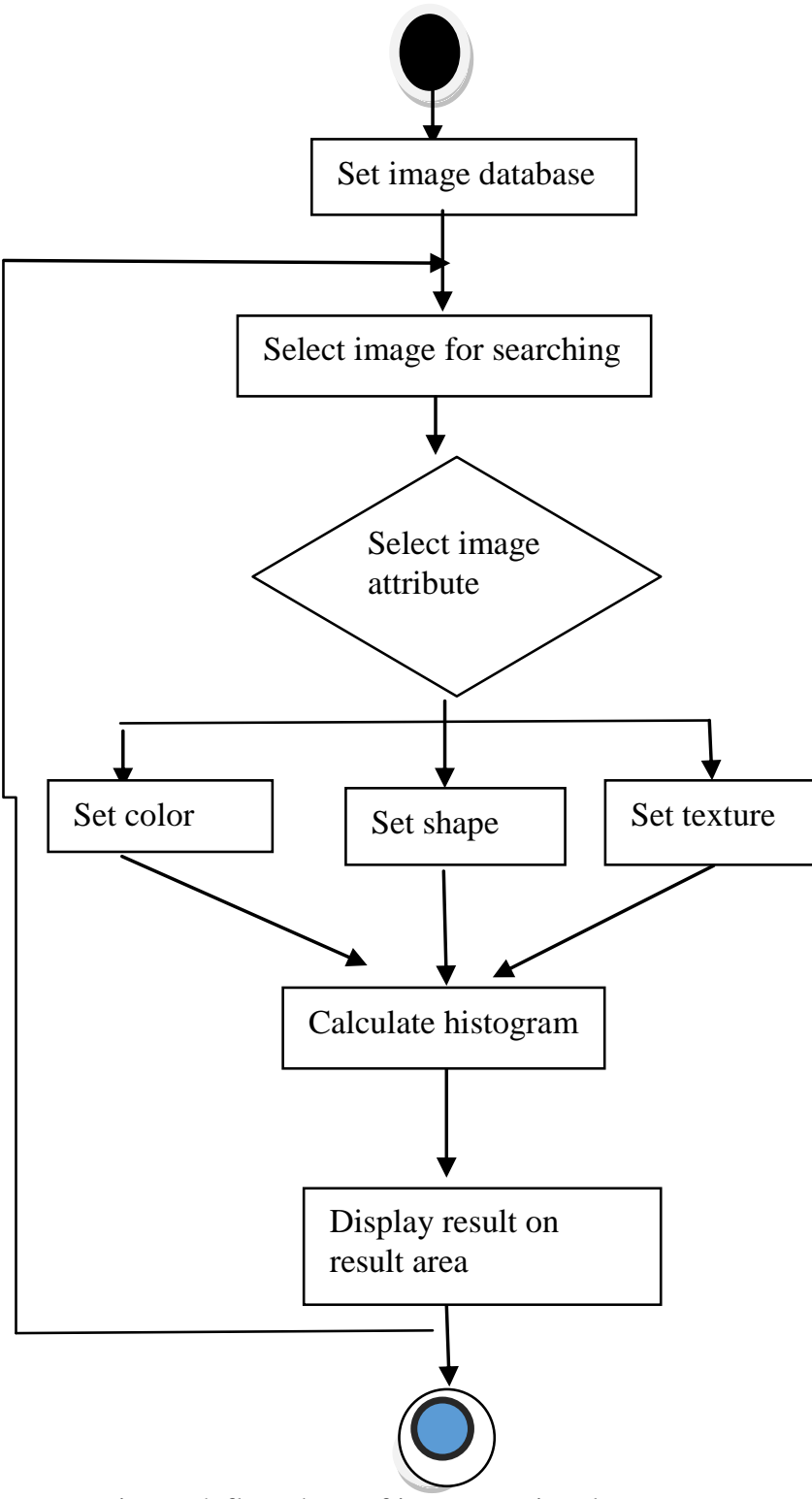

Figure 4-flowchart of image retrieval system

Colour attribute: - Here pixel by pixel histogram of a query image plotted and histogram matched with database images. Two different images of different types can have same color histogram.

Shape attribute: - Shape wise histogram of a query image plotted. Here edges are taking into consideration. Here two images of different colours but having the same shapes can have same shape histogram.

Texture attribute: - Here pixel by pixel histogram of a texture part of image plotted. Images that can have same part may generate same texture histogram.

\section{CONCLUSION}

This paper shows that, image can be searched by using different type of image. The features of different query images can be extracted and these features are used as attributes. The merit of this paper is associated with the fusion of color, shape and texture. One major difference between our image retrieval system and other image retrieval system, it takes feature embedding images to find the result. The current techniques are based on minimum attributes of an image. Further in future it can be improve by using maximum attributes. The best result where obtained by combining multiple attributes.

\section{REFERENCES}

[1] Pallavi Bhatt, Pradeep Rusiya, Vijay Birchha, "WAGBIR: Wavelet and Gabor Based Image Retrieval Technique for the Spatial-color and Texture Feature Extraction Using BPN in Multimedia Database”, 2014 Sixth International Conference on Computational Intelligence and Communication Networks

[2] "Fusion of Colour, Shape and Texture Features for Content Based Image Retrieval", Pratheep Anantharatnasamy, Kaavya Sriskandaraja, Vahissan Nanda kumar and Sampath Deegalla Department of Computer Engineering, Faculty of Engineering, University of Peradeniya, Sri Lanka

[3] K.Jayanthi, Dr.M.Karthikeyan, "Efficient Fuzzy color and Texture Feature Extraction Technique for Content Based Image Retrieval System", ECE Department, Tamilnadu College of Engineering, Coimbatore, India

[4] MarekBleschke, RafelMadonski, RadoslawRudnicki, "Image Retrieval System Based on Combined MPEG-7 Texture and Colour Descriptors", MIXDES 2009, 16th International Conference "Mixed Design of Integrated Circuits and Systems", June 25-27, 2009, Poland

[5] Kamlesh Kumar, Zain-ul-abiding, Jian-Ping Li, Riau Ahmed Shaikh, "Content Based Image Retrieval Using Gray Scale Weighted Average Method", (IJACSA) International Journal of Advanced Computer Science and Applications, Vol. 7, No. 1, 2016

[6] Tarun Kumar, Karun Verma, "A Theory Based on Conversion of RGB image to Gray Image", International Journal of Computer Applications (0975 - 8887) Volume 7- No.2, September 2010

[7] RitikaHirwane,"Shape Base Image Retrieval”, International Journal of Advanced Research in Computer and Communication Engineering Vol. 4, Issue 12, December 2015

[8] Sangoh Jeong, "Histogram-Based color Image Retrieval", Psych221/EE362 Project Report Mar.15, 2001

[9] Greg Pass RaminZabih "Comparing Images Using Joint Histograms", Computer Science Department Cornell University Ithaca, NY 14853

[10] ChestiAltaffHussain, Dr. D. Venkata Rao, T. Praveen, "COLOR HISTOGRAM BASED IMAGE RETRIEVAL”, Hussain, et al., International Journal of Advanced Engineering Technology

[11] Allan Hanbury, Beatriz Marcotegui, "Colour Adjacency Histograms for Image Matching”, PRIP, Institute of ComputerAided Automation, Vienna University of Technology, Favoritenstraße 9/1832, A-1040 Vienna, Austria

[12] Ch.Kavitha, Dr.B.Prabhakara Rao, Dr.A.Govardhan, "Image Retrieval Based On color and Texture Features of the Image Subblocks", International Journal of Computer Applications (0975 8887) Volume 15- No.7, February 2011

[13] P. JemimahJebaPriya, T. Nalini and M. Padmavathy, "Bi-Similarity Mapping Based Image Retrieval Using Shape Features", International journal of application or Innovation in engineering and management Volume 2, Issue 4, April 2013

[14] Kiran Kumar K, Ramyakrishna I, Mary Santhi Sri T, VamshiPriya V, Avinash Reddy G, "Image Based Retrieval Using Edge Detection Algorithm" International Journal \& Magazine of Engineering, Technology, Management and Research A Peer Reviewed Open Access International Journal

[15] IN-WU CHEN, GUANG-LI YU , JUN-BIN GONG , "TEXTURE IMAGE RETRIEVAL SYSTEM", Proceedings of the 2010 International Conference on Wavelet Analysis and Pattern Recognition, Qingdao, 11-14 July 2010

[16] Rahul Mehta, Nishchol Mishra, SanjeevSharma "color - Texture based Image Retrieval System", International Journal of Computer Applications (0975 - 8887) Volume 24- No.5, June 201124

[17] Yossi Rubner and Carlo Tomasi "Texture-Based Image Retrieval without Segmentation", Computer Science Department Stanford University Stanford, CA 94305 